
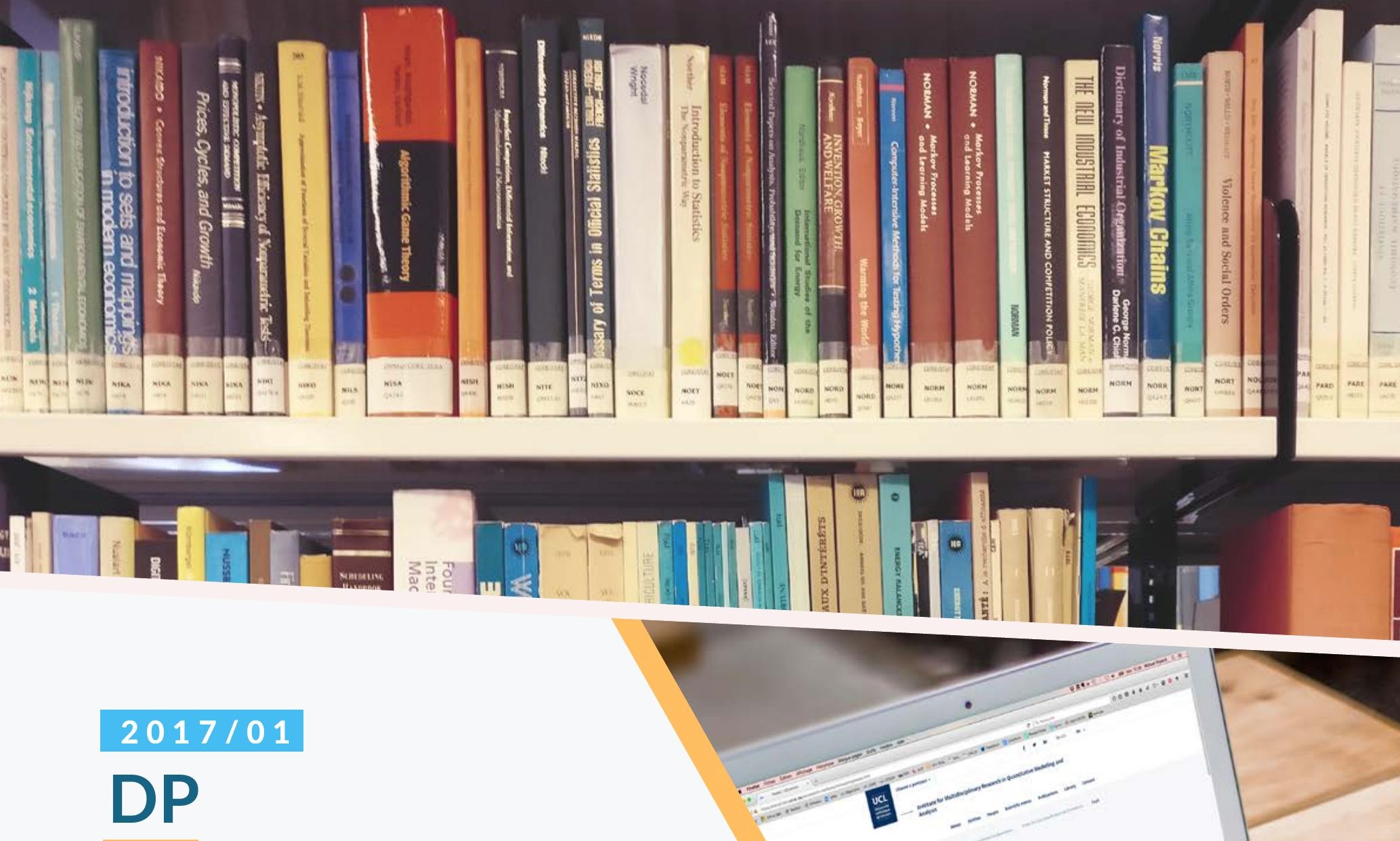

Matias Schuster, Stefan Minner and Jean-Sébastien Tancrez

Two-stage supply chain design with safety stock placement decisions

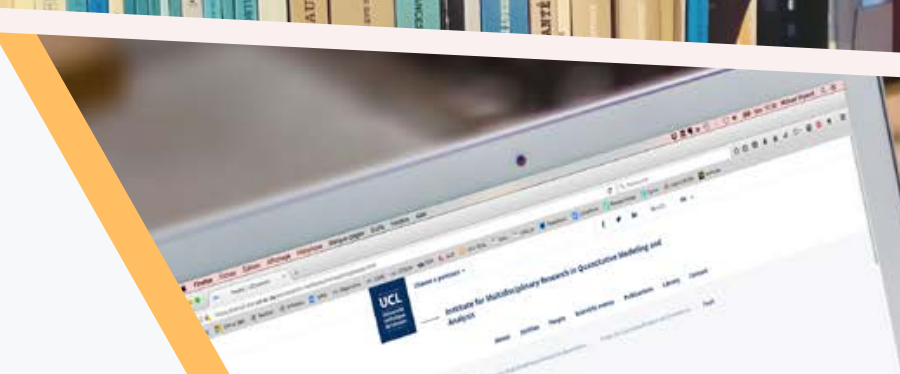




\section{CORE}

Voie du Roman Pays 34, L1.03.01

Tel (32 10) 474304

Fax (32 10) 474301

Email: immaq-library@uclouvain.be

http://www.uclouvain.be/en-44508.html 


\title{
Two-stage supply chain design with safety stock placement decisions
}

\author{
Matias Schuster ${ }^{\mathrm{a}, *}$, Stefan Minner ${ }^{\mathrm{b}}$, Jean-Sébastien Tancrez ${ }^{\mathrm{a}}$ \\ ${ }^{a}$ CORE, Louvain School of Management, Université catholique de Louvain. \\ ${ }^{b}$ TUM School of Management, Technische Universität München.
}

\begin{abstract}
In this paper, we propose a supply chain design model that integrates facility location with safety stock placement and delivery strategy decisions, to reflect their interdependence and ultimately improve the resulting supply chain design. Safety stock placement decisions are presented as an extended version of the guaranteed-service model, with differentiated service times quoted from distribution centres to retailers. We also consider two customer classes that differ with respect to their delivery time preferences at the retailers. The resulting non-linear model is formulated as a conic quadratic mixed-integer program, which can be solved to optimality. The computational experiments capture the trade-offs between location and safety stock placement decisions, between demand variability pooling and proximity to retailers, and between lead times and service times. We show that, when the distribution centres have short lead times to the retailers, both of them tend to hold safety stocks, and the retailers offer express deliveries. Conversely, when lead times are long, only the retailers hold safety stocks and they tend to offer regular deliveries.
\end{abstract}

Keywords: Location-Inventory; Safety Stock Placement; Risk Pooling; Service Times;

Customer Classes.

\footnotetext{
*This research was partly conducted while the first author was a visiting PhD student at the Technical University of Munich. Financial support from the Belgian Fund for Scientific Research (FNRS) through the FRESH grant is gratefully acknowledged.
} 


\section{Introduction}

Supply chain management provides an effective way to increase competitive advantage by reducing operational costs and improving customer's satisfaction. A critical decision that impacts the performance of a company in the long term is the strategic design of its supply chain. Facilities are a key driver of supply chain performance in terms of responsiveness and efficiency. On the one hand, companies can gain economies of scale when centralizing products in one location, which leads to an increase in efficiency. On the other hand, this efficiency increase comes at the expense of responsiveness, as many of the customers may be located far from the facilities (Chopra and Meindl [2016]). Moreover, in today's volatile economy, demand uncertainty is a major concern for companies, and it forces them to hold safety stocks. Therefore, the optimal placement of safety stocks is an important decision when designing the supply chain as it has a direct impact on the service quality. The decisions on where to locate facilities and where to place the safety stocks in these facilities are interdependent by nature. In this context, the integration of these decisions leads to a more efficient design of the supply chain.

In this paper, we propose a mathematical model that integrates safety stock placement decisions into the supply chain design problem. We consider two-stage supply chains composed of retailers, distribution centres (DCs) and one central plant. Our model simultaneously determines: (i) the number and location of opened DCs, (ii) the allocation of flows between DCs and retailers, (iii) for which retailers the opened DCs hold safety stocks, and (iv) the delivery time options at retailers. We use the guaranteed-service approach to model the safety stock placement decisions between DCs and retailers. The guaranteed-service model assumes that opened DCs serve each retailer with a deterministic service time, which is defined as the time elapsed between an order placed at the DC and the release of that order to the retailer. Several trade-offs are captured by integrating safety stock placement decisions into the strategic supply chain design problem. On the one hand, when safety stocks are only held at retailers, the lead time is assumed to be the delivery time between the central plant and the retailers. Consequently, the company can benefit from the lead 
time pooling effect in certain situations (e.g., for long lead times between DCs and the retailers, and short order lead times between the central plant and the DCs). On the other hand, when safety stocks are held at DCs, the company can reduce safety stock costs by pooling demand variability at DCs (see Hua and Willems [2015]). Therefore, interesting managerial insights arise from the balance between lead time and demand variability pooling strategies, which are influenced by the number of opened DCs, the safety stock placement and the delivery strategies.

Moreover, we differentiate customers at retailers regarding their delivery time preference. Customers like to be able to choose between different delivery options with different price points (Ray and Jewkes [2004], Teimoury et al. [2011]). The degree of flexibility at which a delivery system can meet particular customer needs can influence the decision of customers to place orders, and thus can be regarded as an important factor in attracting and retaining customers (Novich [1990], Gunasekaran et al. [2004]). In this paper, we include this differentiation and consider two classes of customers. Regular customers at retailers are served with a given service time. To improve its responsiveness, the company also offers express deliveries for those customers that request to be served immediately and accept to pay an extra fee. The income originated by the extra fee of express deliveries will balance the safety stock cost increase caused by the improvement of the delivery time. Interesting managerial insights are revealed when we consider different customer classes in a supply chain design model. The trade-offs arise from the selection of express and regular deliveries, which are dependant on the lead times, the express deliveries demand, the service times and the fees. In particular, when considering short lead times between DCs and the retailers, express deliveries are offered more often as safety stock costs are limited.

The main contributions of this paper are summarized as follows. Firstly, we propose a location-inventory model with demand uncertainty that integrates safety stock placement and delivery time decisions. Secondly, we extend the guaranteed-service model by considering different service times between DCs and the retailers, and different classes of customers. Thirdly, the resulting model is reformulated as a conic quadratic mixed-integer program (CQMIP), which can be solved efficiently using standard optimization software packages 
such as CPLEX. Finally, to show the benefits of integrating facility location and safety stock placement decisions, we conduct a large set of computational experiments. We derive interesting managerial insights related to the impact of service times and lead times on the placement of safety stocks and on the delivery options selected.

The paper is organized as follows. The next section reviews the existing literature on supply chain design and strategic safety stock placement. In Section 3, we define our problem in detail and give the CQMIP formulation. In Section 4, we show the results of the computational experiments and discuss managerial insights. We conclude in Section 5 and outline ideas for future work.

\section{Literature review}

Our research is grounded in the literature on the location-inventory and on the safety stock placement stream. In this section, we give a detailed review on each field, and on the papers that combine both streams, which are the closest to our research.

For a detailed review on the facility location stream, we refer the reader to Owen and Daskin [1998], ReVelle and Eiselt [2005], Klose and Drexl [2005], and Bravo and Vidal [2013]. During the last 15 years, the basic location models have been generalized by integrating tactical and operational decisions. We refer the reader to Beamon [1998, Shen [2007], Melo et al. [2009], Mula et al. [2010], and Farahani et al. [2015] for a review on different integrated models such as location-inventory, location-routing or inventory-routing models.

We focus on the integrated location-inventory problem, and we consider safety stocks for capturing demand uncertainty. The number of stocking locations has long been proven to directly affect the total amount of inventory held at these locations. Smykay 1973 notes that the safety stock held at a centralized location is equal to the aggregate safety stock held at multiple locations divided by the square root of the number of locations (Evers [1995]). Eppen [1979] compares a decentralized system in which a separate inventory is held at each retailer with a centralized one in which inventory is held at a DC. His model demonstrates that, due to the risk pooling effect, the consolidation of demand can reduce holding and penalty costs. In accordance with this feature, Shen et al. 2003 propose a 
location-inventory model with risk pooling. Their model determines the location of DCs, the allocation of flows, how often to reorder and which level of safety stock to maintain at DCs in order to minimize costs (i.e., opening, transportation and inventory). Computational results show that centralizing stocks at DCs leads to inventory reductions via risk pooling. Daskin et al. 2002] solve the same location-inventory problem using Lagrangian relaxation, for instances with up to 150 customers. In a similar fashion, Miranda and Garrido [2004] combine a capacitated facility location problem with safety stocks. The resulting non-linear mixed-integer problem is solved using Lagrangian relaxation and sub-gradient methods. $\mathrm{Ku}-$ mar and Tiwari 2013 show the benefits of risk pooling in a three-layer supply chain and compare the savings from a centralized system with those from a decentralized one. Sourirajan et al. 2009 solve a location-inventory model using genetic algorithms and show the trade-off between lead times and inventory risk pooling. Park et al. [2010] formulate the location-inventory problem as a nonlinear integer programming model, for which a twophase heuristic solution algorithm is derived based on the Lagrangian relaxation approach. Atamtürk et al. 2012] study the location-inventory problem for different cases such as capacitated facilities, correlated demands and multicommodities. Their main contribution relies on the formulation of these models as conic quadratic mixed-integer programs (CQMIPs). Shahabi et al. 2013] also reformulate the location-inventory model as a compact CQMIP for a four-echelon supply chain where warehouses and hubs are located, and where safety stocks are held at warehouses. Schuster and Tancrez 2016 formulate the location-inventory problem as a non-linear continuous model, which is solved using an heuristic algorithm, allowing the design of large supply chains. In their model, safety stocks are held at DCs and at retailers.

The strategic safety stock placement problem has been widely studied in the last 20 years. We refer the reader to Minner [2000] for a detailed presentation of the fundamental principles of safety stock planning. Graves and Willems 2003 give a review of the two general models for locating safety stocks: the stochastic service model and the guaranteed-service model. The two approaches differ in their demand treatment and service time characterization. In the stochastic model, the retailers may suffer stochastic delays when the DC does not have 
enough items available to deliver. Therefore, the service time is stochastic. In contrast, in the guaranteed-service model, the service time is a deterministic decision variable. The guaranteed-service approach can simplify the safety stock placement problem as it can be formulated as a deterministic mathematical program. In a recent survey, Eruguz et al. 2016 give a detailed review on guaranteed-service models for multi-echelon inventory optimization.

In this paper, we use the guaranteed-service approach to model the safety stock placement decisions. The guaranteed-service model is first introduced by Simpson [1958], who models a serial chain production as a sequence of in-process inventories. The objective of the model is to determine the inventory service time at each production stage in order to satisfy the demand of its downstream stage at minimum inventory costs. Under this serial network structure, the paper shows that the minimum cost occurs when the inventory at each stage is either full or empty. Since then, the basic model has been extended in different ways. Inderfurth and Minner 1998 formulate the safety stock placement problem without internal delays and show that the optimal policy depends on the structure of the multi-stage system and the service measure used. Hua and Willems [2015 use the guaranteed-service model in a two-stage serial supply chain to give analytical insights on the optimal safety stock placement policy with regard to supply chain costs and lead times. Instead of optimizing the placement of safety stocks, Chen and Li [2015] use the guaranteed-service approach to optimize the (R,Q) inventory policy for a serial supply chain with Poisson demand and fixed order costs. The model is solved by an iterative procedure using dynamic programming. $\mathrm{Hu}-$ mair et al. 2013 consider stochastic demand and lead times for the safety stock placement optimization problem. Osman and Demirli 2012 also consider uncertainty in demand and lead time. They propose a decentralized and a centralized safety stock placement model. The decentralized model is solved using a non-linear commercial solver, whereas a Benders decomposition technique is developed to solve the centralized model. Grahl et al. 2014 solve the safety stock placement problem with service time differentiation using metaheuristics in general acyclic networks. Li and Jiang [2012] also model the safety stock placement problem in general acyclic networks. The problem is solved by integrating constraint programming into a genetic algorithm. Magnanti et al. [2006] solve the safety stock placement 
problem using a commercial solver that generates flow cover cuts by considering redundant constraints.

Only few papers integrate safety stock placement decisions and supply chain design, as we aim to do in this paper. Graves and Willems 2005 incorporate multi-echelon stock placement into a network optimization problem for spanning-tree type supply chains with stationary demand. The supply chain design problem consists of the selection of the best option at each stage in terms of lead times and direct costs. They solve this problem using a dynamic programming heuristic algorithm. Following a similar approach, Funaki 2012 formulates a multi-echelon stock placement problem as a modification of the guaranteedservice model with due-date based and non-stationary demand. The optimization procedure is also based on dynamic programming. Moncayo-Martínez and Zhang [2013 formulate the problem of supply chain design and safety stock placement as a Max-Min system. Their solution approach is based on ant colony optimization to minimize a bi-objective function composed by the total cost (safety stock and production costs) and the lead times, to ensure product deliveries without delays. These papers relate supply chain design and safety stock placement but suppose that the facilities are existing and fixed. The combination of facility location and safety stock placement decisions is barely included in the literature. You and Grossmann 2010 consider fixed opening costs of DCs, and use expected lead times to quantify responsiveness of a three-echelon supply chain. The authors solve the model by using a spatial decomposition algorithm based on the integration of Lagrangian relaxation and piecewise linear approximation for cases up to 15 plants, 100 potential DC locations and 200 customers in around 10 hours. This work is extended by Rodriguez et al. [2014] with the consideration of lost sales to redesign the supply chain of spare part delivery. They solve the redesign problem for electric motor supply chains.

Compared to these papers, and in particular to You and Grossmann 2010] and to Rodriguez et al. [2014], our research differs in several aspects. First, our model integrates features that are not combined in the cited papers such as differentiated service times from DCs to retailers and customer classes depending on their delivery time preference. Second, we avoid the use of continuous service times variables between DCs and the retailers to 
model the safety stock placement decisions. Instead, we include binary variables to explicitly decide for which connected retailers the opened DCs hold safety stocks. Finally, our location-inventory model with safety stock placement decisions is formulated as a CQMIP, which is efficiently solved to optimality.

\section{Location-Inventory model with safety stock placement decisions}

\subsection{Model presentation}

In this section, we explain our model in detail. We consider two-stage supply chains with single sourcing and a single product. We assume that the vehicles have a given capacity, and consider full truckloads between DCs and retailers. The central plant has unlimited production capacity. The locations of the retailers are known and fixed. We suppose that retailers' demands are uncorrelated and normally distributed, with a known mean and variance. The lead times between potential DCs and retailers are assumed to be known and dependent on the distance. At DCs, the order lead times to the central plant are also known.

In our model, two different delivery options can be followed at retailers, depending on whether express services are offered or not. When the first delivery option is applied, the retailers offer the possibility to customers to have their product immediately (the product is available in stock). Therefore, a given percentage $\left(P_{r}^{E D}\right)$ of the customers at retailer $r$ choose this option and is served immediately, with express deliveries, while the rest of the customers $\left(1-P_{r}^{E D}\right)$ is served with regular deliveries (with a given service time, $S_{r}$ ). The customers served with express deliveries accept to pay an additional fee $\left(T_{r}\right)$ for the service. In the second delivery option, the retailer $r$ does not propose express deliveries, so all the customers are served with regular deliveries (with the given service time, $S_{r}$ ).

Furthermore, our model includes cycle as well as safety inventory. On the one hand, cycle inventory is computed at retailers and at DCs. The cycle inventory at retailers depends on the vehicles' capacity between DCs and the retailers. At DCs, the cycle inventory is determined by the size of the order coming from the central plant, which is found by balancing ordering and inventory costs. On the other hand, safety stocks are included to 


\begin{tabular}{cccc}
\hline \multicolumn{4}{c}{ Safety Stock Placement Strategies } \\
\hline \multirow{4}{*}{ Strategy } & DC & Retailer & Description \\
\cline { 2 - 3 } 1 & - & $L T_{d}+L T_{r}^{d}$ & Safety Stocks are held at retailers \\
2 & $L T_{d}$ & $L T_{r}^{d}$ & Safety Stocks are held at DCs and at retailers \\
\hline
\end{tabular}

Table 1: The net lead time at DCs and at retailers for each safety stock placement strategy.

absorb demand uncertainty. The safety stock placement decisions are modelled using the guaranteed-service approach. As already mentioned in the previous section, Simpson 1958 shows that, under certain assumptions, safety stock inventories are either full or empty for two-stage supply chains. This result leads to two different safety stock placement strategies, depending on whether safety stocks are held at DCs or not. It also allows us to avoid the use of continuous service time variables between DCs and the retailers, as instead we can use binary variables that directly determine whether one strategy or the other is used for each retailer.

The net lead time, defined as the time during which safety stocks are required to cover demand variation, differs for the two strategies. The net lead times, at DCs and at retailers, are given for each strategy in Table 1, and are explained in the following. In safety stock placement strategy one, the DCs do not hold safety stocks and thus the net lead times at DCs are not relevant. The net lead time at a retailer $r$ corresponds to the time from the central plant to the retailer, which is given by the order lead time from the central plant to the linked DC $d, L T_{d}$, plus the lead time between DC $d$ and retailer $r, L T_{d r}$. In safety stock placement strategy two, the DCs and the retailers hold safety stocks. At a DC $d$, the net lead time corresponds to the time from the central plant to $\mathrm{DC} d, L T_{d}$. At a retailer $r$, the net lead time is given by the lead time between the linked DC $d$ and the retailer $r, L T_{d r}$. Note that when regular deliveries are applied at retailer $r$, the service time $S_{r}$ of regular deliveries is subtracted, and the net lead time is given by $L T_{d}+L T_{r}^{d}-S_{r}$ for strategy one, and by $L T_{r}^{d}-S_{r}$ for strategy two. 


\subsection{Notation}

In the following, we introduce the notation used throughout this paper.

\section{Indices and sets:}

$r \in R=\left\{1, \ldots, n_{r}\right\}$ for retailers,

$d \in D=\left\{1, \ldots, n_{d}\right\}$ for potential DC locations,

$p \in P=\{1,2\}$ for safety stock placement strategies (1, for safety stocks only at the retailer;

2 , for safety stocks at the retailer and at the DC).

\section{Parameters:}

$O_{d r}$ : unit transportation cost from DC $d$ to retailer $r$, in $\$ /$ item,

$C_{d r}$ : capacity of vehicles from DC $d$ to retailer $r$, in items/vehicle,

$K_{d}$ : fixed cost at DC $d$ of placing an order to the central plant, in $\$ /$ order,

$F_{d}$ : fixed cost of opening a DC $d$, in $\$ /$ period,

$h_{r}$ : unit inventory holding cost at retailer $r$, in $\$ /($ item $\cdot$ period $)$,

$H_{d}$ : unit inventory holding cost at $\mathrm{DC} d$, in $\$ /($ item $\cdot$ period $)$,

$T_{r}$ : additional/expected fee per unit applied to the express customers of retailer $r$, in $\$ /$ item,

$z_{\alpha}:$ standard normal deviate associated with service level $\alpha$,

$L T_{d}$ : order lead time between the central plant and the DC $d$, in periods,

$L T_{d r}$ : lead time between the DC $d$ and the retailer $r$, in periods,

$S_{r}$ : Service time of regular deliveries at retailer $r$, in periods,

$\mu_{r}$ : mean demand at retailer $r$, in items/period,

$P_{r}^{E D}$ : percentage of customers at retailer $r$ that request express deliveries $E D$, in $\%$,

$\sigma_{r}^{E D}$ : standard deviation of express deliveries' demand $E D$ at retailer $r$, in items/period,

$\sigma_{r}^{R D}$ : standard deviation of regular deliveries' demand $R D$ at retailer $r$, in items/period,

$\sigma_{r}$ : standard deviation of total demand at retailer $r$, in items/period,

$C V_{r}^{E D}$ : coefficient of variation of express deliveries' demand $E D$ at retailer $r$,

$C V_{r}^{R D}$ : coefficient of variation of regular deliveries' demand $R D$ at retailer $r$.

\section{Decision variables:}

$y_{d}$ : equals one when $\mathrm{DC} d$ is opened, zero otherwise,

$x_{d r}^{p}$ : equals one when DC $d$ serves retailer $r$ using safety stock placement strategy $p$, zero 
otherwise,

$q_{d}$ : size of order to the central plant at DC $d$, in items,

$w_{r}^{E D}$ : equals one when retailer $r$ proposes express deliveries, zero otherwise,

$\lambda_{d}$ : total expected product flow through $\mathrm{DC} d$, in items/period,

$\bar{\sigma}_{d}$ : standard deviation of the product flow through DC $d$, in items/period.

\subsection{Cost function}

In this section, we describe the various costs included in our model. Our mathematical model minimizes transportation, facility opening, cycle inventory, ordering and safety stock costs. The transportation cost between a DC $d$ and a retailer $r$ is computed as the cost per unit, $O_{d r}$, times the retailer's demand, $\mu_{r}$, and the allocation decision variable, $x_{d r}^{p}$. It leads to:

$$
\sum_{d \in D, r \in R, p \in P} O_{d r} \cdot \mu_{r} \cdot x_{d r}^{p}
$$

Next, the objective function includes the fixed opening cost of DCs. It can be written as:

$$
\sum_{d \in D} F_{d} \cdot y_{d}
$$

The cycle inventory cost at retailer $r$ is computed with the unit inventory cost $h_{r}$, times the average inventory level. The latter equals half the vehicle's capacity, $C_{d r} / 2$, times the allocation decision variable, $x_{d r}^{p}$. The cycle inventory cost at retailers is given by:

$$
\sum_{d \in D, r \in R, p \in P} h_{r} \cdot \frac{C_{d r}}{2} \cdot x_{d r}^{p}
$$

We note that if the unit holding cost is the same for all retailers, and the vehicle's capacity is the same for each link between DCs and retailers, the cycle inventory cost is constant and does not impact the optimal decisions.

The cycle inventory cost at DC $d$ is simply computed as the unit holding cost, $H_{d}$, times the average inventory level, $q_{d} / 2$. The order cost is given by the cost of placing an order, 
$K_{d}$, times the number of orders executed per period, $\sum_{r \in R, p \in P} \mu_{r} \cdot x_{d r}^{p} / q_{d}$. Thus, the ordering and cycle inventory costs are:

$$
\sum_{d \in D} H_{d} \cdot \frac{q_{d}}{2}+\sum_{d \in D} K_{d} \cdot \frac{\sum_{r \in R, p \in P} \mu_{r} \cdot x_{d r}^{p}}{q_{d}}
$$

The order size variable can be computed as an EOQ, leading to $q_{d}=\sqrt{2 \cdot K_{d} \cdot \sum_{r \in R, p \in P} \mu_{r} \cdot x_{d r}^{p} / H_{d}}$. The holding and order costs thus become:

$$
\sum_{d \in D} \sqrt{2 \cdot H_{d} \cdot K_{d} \cdot \sum_{r \in R, p \in P} \mu_{r} \cdot x_{d r}^{p}}
$$

Note that our model could be interpreted as locating factories (without a central plant) instead of locating DCs. In this case, the order cost would correspond to a production setup cost, and the order size to a batch size.

The safety stock costs at retailers are computed by multiplying the holding cost at retailer $r, h_{r}$, the normal standard deviate, $z_{\alpha}$, where $\alpha$ represents the service level (i.e., the probability of satisfying all the demand during the net lead time through safety stocks), and the standard deviation of the demand during the net lead time. The latter depends on the delivery and on the safety stock placement strategies. For regular and express deliveries, the standard deviation $\left(\sigma_{r}\right)$ is multiplied by the allocation decision variables $x_{d r}^{p}$, also determining the safety stock placement strategy $p$, and then by the square root of the corresponding net lead time, showed in Table 1. Moreover, when the retailers offer express deliveries $\left(w_{r}^{E D}=1\right)$ to their customers, safety stocks should also cover the service time, $S_{r}$, which is not covered by the previous safety stock. Consequently, the safety stock costs at retailers are determined by:

$\sum_{r \in R} h_{r} \cdot z_{\alpha} \cdot\left(\sigma_{r} \cdot\left[\sum_{d \in D}\left(x_{d r}^{1} \cdot \sqrt{L T_{d}+L T_{d r}-S_{r}}+x_{d r}^{2} \cdot \sqrt{L T_{d r}-S_{r}}\right)\right]+\sigma_{r}^{E D} \cdot w_{r}^{E D} \cdot \sqrt{S_{r}}\right)$

The demand of customers is composed of express and regular deliveries. Therefore, the standard deviation is $\sigma_{r}^{E D}=C V_{r}^{E D} \cdot \mu_{r} \cdot P_{r}^{E D}$ for express deliveries demand, and $\sigma_{r}^{R D}=$ 
$C V_{r}^{R D} \cdot \mu_{r} \cdot\left(1-P_{r}^{E D}\right)$ for regular deliveries demand. Consequently, the standard deviation of all demands, express and regular, is $\sigma_{r}=\sqrt{\left(\sigma_{r}^{E D}\right)^{2}+\left(\sigma_{r}^{R D}\right)^{2}}$.

When safety stock placement strategy two is selected, safety stocks are held at retailers as well as at DCs. The safety stock costs at DCs are computed by multiplying the holding cost at DCs, $H_{d}$, the normal standard deviate, $z_{\alpha}$, and the standard deviation of the demand during the order lead time between the central plant and the DC $d, \sqrt{L T_{d} \cdot \sum_{r \in R} \sigma_{r}^{2} \cdot x_{d r}^{2}}$. The safety stock cost at DCs is thus given by:

$$
\sum_{d \in D} H_{d} \cdot z_{\alpha} \cdot \sqrt{L T_{d} \cdot \sum_{r \in R} \sigma_{r}^{2} \cdot x_{d r}^{2}}
$$

Finally, when retailers offer express deliveries $\left(w_{r}^{E D}=1\right)$ an additional fee is charged to the customers, which results in an income for the company. It is computed by multiplying the extra fee per product, $T_{r}$, and the express deliveries demand, given by $P_{r}^{E D} \cdot \mu_{r}$. The total income originated by the extra fees is determined by:

$$
\sum_{r \in R} P_{r}^{E D} \cdot \mu_{r} \cdot T_{r} \cdot w_{r}^{E D}
$$

\subsection{CQMIP formulation}

In this subsection, we formulate our model as a conic quadratic mixed-integer program. We replace the non-linear terms in 5 and 7 with auxiliary variables $\lambda_{d}$ and $\bar{\sigma}_{d}$, and include the following quadratic constraints using the fact that $x_{d r}^{p}=\left(x_{d r}^{p}\right)^{2}$ :

$$
\begin{array}{ll}
\sum_{r \in R, p \in P} \mu_{r} \cdot\left(x_{d r}^{p}\right)^{2} \leq \lambda_{d}^{2} & \forall d \in D, \\
\sum_{r \in R} \sigma_{r}^{2} \cdot\left(x_{d r}^{2}\right)^{2} \leq \bar{\sigma}_{d}^{2} & \forall d \in D .
\end{array}
$$


The location-inventory model with safety stock placement decisions and delivery strategies is formulated by:

$$
\begin{aligned}
& \min \sum_{d \in D, r \in R, p \in P} O_{d r} \cdot \mu_{r} \cdot x_{d r}^{p} \\
& +\sum_{d \in D} F_{d} \cdot y_{d} \\
& +\sum_{d \in D, r \in R, p \in P} h_{r} \cdot \frac{C_{d r}}{2} \cdot x_{d r}^{p} \\
& +\sum_{d \in D} \sqrt{2 \cdot H_{d} \cdot K_{d}} \cdot \lambda_{d} \\
& +\sum_{r \in R} h_{r} \cdot z_{\alpha} \cdot\left(\sigma_{r} \cdot\left[\sum_{d \in D}\left(x_{d r}^{1} \cdot \sqrt{L T_{d}+L T_{d r}-S_{r}}+x_{d r}^{2} \cdot \sqrt{L T_{d r}-S_{r}}\right)\right]+\sigma_{r}^{E D} \cdot w_{r}^{E D} \cdot \sqrt{S_{r}}\right) \\
& +\sum_{d \in D} H_{d} \cdot z_{\alpha} \cdot \sqrt{L T_{d}} \cdot \bar{\sigma}_{d} \\
& -\sum_{r \in R} P_{r}^{E D} \cdot \mu_{r} \cdot T_{r} \cdot w_{r}^{E D} \\
& \text { s.t } \sum_{r \in R, p \in P} \mu_{r} \cdot\left(x_{d r}^{p}\right)^{2} \leq \lambda_{d}^{2} \quad \forall d \in D, \\
& \sum_{r \in R} \sigma_{r}^{2} \cdot\left(x_{d r}^{2}\right)^{2} \leq \bar{\sigma}_{d}^{2} \quad \forall d \in D, \\
& \sum_{d \in D, p \in P} x_{d r}^{p}=1 \quad \forall r \in R, \\
& x_{d r}^{p} \leq y_{d} \quad \forall r \in R, d \in D, p \in P \\
& \lambda_{d}, \bar{\sigma}_{d} \geq 0 \quad \forall d \in D, \\
& w_{r}^{E D}, x_{d r}^{p}, y_{d} \in\{0,1\} \quad \forall r \in R, d \in D, p \in P .
\end{aligned}
$$

The objective function includes, respectively, the transportation cost (11); the facility opening cost (12); the inventory cost at retailers (13); the order cost at DCs (14); the safety stock cost (15)-(16) and the income from additional fees (17). Constraints (18)-(19) are the quadratic constraints defining the auxiliary variables. Constraints 20 ensure that each retailer is assigned to one DC using one safety stock placement strategy. Constraints 21) guarantee that the retailers are linked to opened DCs. Finally, constraints 22 give the non- 
negativity constraints of the auxiliary variables and (23) define the domain of the binary decision variables.

The main advantages of the CQMIP approach is that it is direct, efficient and flexible, as it can be solved using standard optimization software packages without the need of specialized algorithms (Atamtürk et al. 2012]). Standard optimization software packages such as CPLEX offer the possibility of solving CQMIPs based on interior-point methods and branch and bound algorithms. However, the performance of the branch and bound algorithm can be improved by strengthening the formulations with cutting planes. Therefore, we have reformulated the quadratic constraints $(18)-19$ with polymatroid inequalities to strengthen the convex relaxation of the CQMIP. The separation problem for the extended polymatroid inequalities is solved by the greedy algorithm of Edmonds [1970]. We refer the reader to Atamtürk and Narayanan [2008] and to Atamtürk et al. [2012 for a detailed explanation of the separation problem and the greedy algorithm.

\section{Numerical experiments}

In this section, we present computational experiments to illustrate the application of our approach and obtain managerial insights.

\subsection{Computational results}

We use the data from the 1990 U.S Census given in Daskin 1995. We employ the 88-node data set, which reports data of the lower U.S state capitals plus Washington DC and the 50 largest U.S cities. In Table 2, we summarize the values used for the different parameters. The potential DC locations are chosen to be the retailers' locations $\left(n_{d}=n_{r}\right)$. The retailer's demand, $\mu_{r}$, is taken by dividing the population of each city by 1,000 (as in Atamtürk et al. [2012]). Transportation unit costs, $O_{d r}$, are proportional to the distances between DCs and the retailers, with a unit distance cost of $0.001 \$ / \mathrm{km}$. The lead times between DCs and the retailers, $L T_{d r}$, are computed based on a fixed time $\left(L T_{d r}^{\text {Fixed }}\right)$ that varies between 0 and 20 days, plus a variable time that depends on the distance (assuming an average vehicle speed of $60 \mathrm{~km} / \mathrm{h}$ ). We assume that the order lead times between the 


\begin{tabular}{ll}
\hline Parameters & Values \\
\hline Indexes & \\
$n_{r}=n_{d}$ & 88 \\
Network parameters & $(1,5)$ days \\
$L T_{d}$ & $(0,1,5,10,20)$ days \\
$L T_{d r}^{F i x e d}$ & $60 \mathrm{~km} / \mathrm{h}$ \\
$S p e e d$ & $(20,60,100) \%$ \\
$S_{r}^{\%}$ & \\
Demand parameters & $(0.4,0.8)$ \\
$C V_{r}^{E D}=C V_{r}^{R D}$ & $99 \%$ \\
$\alpha$ & 2.33 \\
$z_{\alpha}$ & $(10,30,50,70,90) \%$ \\
$P_{r}^{E D}$ & \\
Cost parameters & $1000 \$ /$ day \\
$F_{d}$ & $1000 \$ /$ order \\
$K_{d}$ & $0.05 \$ /$ item $\cdot$ day \\
$H_{d}=h_{r}$ & $(0.05,0.25,0.50) \$ /$ item \\
$T_{r}$ &
\end{tabular}

Table 2: Parameter values used for the numerical experiments.

central plant and the DCs, $L T_{d}$, are the same for all DCs, and restricted to being either 1 or 5 days. We compute the vehicle's capacity, $C_{d r}$, as $10 \%$ of the largest retailer's demand. The percentage of express deliveries demand, $P_{r}^{E D}$, is the same at all retailers and varies between 10 and $90 \%$. The service time of regular deliveries, $S_{r}$, which is the same at all retailers, is computed as a percentage (using $S_{r}^{\%}$ ) of $L T_{d r}^{\text {Fixed }}$, the fixed lead time between the DCs and the retailers. The parameter values lead to $2 \cdot 5 \cdot 3 \cdot 2 \cdot 5 \cdot 3=900$ parameter combinations.

The CQMIP is solved in CPLEX on a $2.70 \mathrm{GHz}$ computer with $16 \mathrm{~GB}$ of RAM. Table 3 shows the computational time, the number of polymatroid cuts applied, the number of opened DCs and the optimality gap. The number of opened DCs ranges between 6 and 7 due to the fact that the network parameters (e.g., size of the map, speed) and facility parameters (e.g., facility opening, order and holding costs) are not varied. The CQMIP finds the optimal solution for all instances in an average time of around 15 minutes. Our approach is efficient in solving the location-inventory problem with safety stock placement decisions and delivery strategies. 


\begin{tabular}{|c|c|c|c|c|c|c|c|c|c|}
\hline \multicolumn{3}{|c|}{ Time (Sec.) } & \multicolumn{3}{|c|}{ \# Cuts } & \multicolumn{3}{|c|}{ \# DCs } & \multirow{2}{*}{ Gap (\%) } \\
\hline Min & Avg & Max & Min & Avg & Max & Min & Avg & Max & \\
\hline 546 & 919 & 3103 & 9 & 27 & 70 & 6.00 & 6.44 & 7.00 & 0.00 \\
\hline
\end{tabular}

Table 3: Computational performance of the CQMIP.

\subsection{Managerial insights}

In this section, we derive managerial insights from the computational results. We start by analysing the trade-offs that influence the selection of safety stock placement strategies. In our model, risk pooling has two combined effects that can be inferred from Equations (6) and (7): lead time pooling and demand variability pooling. From Equation (7), we see that demand variability pooling benefits arise for proportionally long order lead times between the central plant and the DCs $\left(L T_{d}\right)$, which will result in the selection of safety stock placement strategy two $\left(x_{d r}^{2}\right)$. On the opposite, when the order lead times between the central plant and the DCs $\left(L T_{d}\right)$ are proportionally short, and the lead times between DCs and the retailers $\left(L T_{d r}\right)$ proportionally long, the demand variability pooling becomes less valuable, leading to choose strategy one $\left(x_{d r}^{1}\right)$ for benefiting from lead time pooling (first term of Equation (6)). These tendencies can be observed in Table 4, where we show the number of retailers served with safety stock placement strategy one or two. Strategy two is more broadly chosen for long order lead times between the central plant and the DCs $\left(L T_{d}\right)$, while strategy one is more frequently selected for long lead times between DCs and retailers $\left(L T_{d r}^{\text {Fixed }}\right)$. We also see in Table 4 that strategy two tends to be selected when the service time $\left(S_{r}^{\%}\right)$ increases, specially if the order lead time between the central plant and the DCs $\left(L T_{d}\right)$ is long. An increase of the service time is equivalent to reducing the lead time between DCs and retailers, increasing the importance of the order lead time at DCs, and therefore the supply chain can benefit from pooling demand variability at DCs. As seen in Table 4 . safety stock placement strategy one tends to be slightly more selected when the coefficient of variation $\left(C V_{r}^{E D}\right)$ increases. Although this may seem contradictory to intuition, it is due to the fact that the number and location of DCs varies when increasing the coefficient of variation. By investigating our results more deeply, we find that when the coefficient 


\begin{tabular}{|c|c|c|c|c|c|c|c|}
\hline \multirow[b]{2}{*}{$C V_{r}^{E D}$} & \multirow[b]{2}{*}{$L T_{d r}^{\text {Fixed }}$} & \multicolumn{2}{|c|}{$S_{r}^{\%}=20$} & \multicolumn{2}{|c|}{$S_{r}^{\%}=60$} & \multicolumn{2}{|c|}{$S_{r}^{\%}=100$} \\
\hline & & $L T_{d}=1$ & $L T_{d}=5$ & $L T_{d}=1$ & $L T_{d}=5$ & $L T_{d}=1$ & $L T_{d}=5$ \\
\hline \multirow{5}{*}{0.4} & 0 & $0 \mid 88$ & $0 \mid 88$ & $0 \mid 88$ & $0 \mid 88$ & $0 \mid 88$ & $0 \mid 88$ \\
\hline & 1 & $15 \mid 73$ & $0 \mid 88$ & $12 \mid 76$ & $0 \mid 88$ & $0 \mid 88$ & $0 \mid 88$ \\
\hline & 5 & $88 \mid 0$ & $9 \mid 79$ & $88 \mid 0$ & $7 \mid 81$ & $0 \mid 88$ & $0 \mid 88$ \\
\hline & 10 & $88 \mid 0$ & $22 \mid 66$ & $88 \mid 0$ & $9 \mid 79$ & $0 \mid 88$ & $0 \mid 88$ \\
\hline & 20 & $88 \mid 0$ & $88 \mid 0$ & $88 \mid 0$ & $22 \mid 66$ & $0 \mid 88$ & $0 \mid 88$ \\
\hline \multirow{5}{*}{0.8} & 0 & $0 \mid 88$ & $0 \mid 88$ & $0 \mid 88$ & $0 \mid 88$ & $0 \mid 88$ & $0 \mid 88$ \\
\hline & 1 & $16 \mid 72$ & $0 \mid 88$ & $13 \mid 75$ & $0 \mid 88$ & $0 \mid 88$ & $0 \mid 88$ \\
\hline & 5 & $88 \mid 0$ & $9 \mid 79$ & $88 \mid 0$ & $7 \mid 81$ & $0 \mid 88$ & $0 \mid 88$ \\
\hline & 10 & $88 \mid 0$ & $29 \mid 59$ & $88 \mid 0$ & $9 \mid 79$ & $0 \mid 88$ & $0 \mid 88$ \\
\hline & 20 & $88 \mid 0$ & $88 \mid 0$ & $88 \mid 0$ & $29 \mid 59$ & $0 \mid 88$ & $0 \mid 88$ \\
\hline
\end{tabular}

Table 4: Number of retailers served with safety stock placement strategy one (on the left) and safety stock placement strategy two (on the right), for different coefficients of variation $\left(C V_{r}^{E D}=C V_{r}^{R D}\right.$ ), service times $\left(S_{r}^{\%}\right)$, order lead times $\left(L T_{d}\right)$ and lead times between DCs and retailers $\left(L T_{d r}^{\text {Fixed }}\right)$. The values in the table are averages over 15 instances (for each value of $P_{r}^{E D}$ and $T_{r}$ ).

of variation increases, DCs are located closer to large cities to mitigate the safety stock increase in these cities. Then, and it is what we observe in our cases, it may happen that a DC located in a new city serves less retailers, which decreases the value of demand variability pooling and leads to a change in the safety stock placement strategy selected (from two to one). This also illustrates the complex and sometimes unintuitive interdependency of the decisions (location, safety stock sizing and placement) and thus the value of an integrated model.

Figure 1 details the supply chain design in four instances, with the number of DCs opened, the allocation of flows between DCs and retailers, and the safety stock placement strategies selected. Several trends can be observed in Figure 1. To begin with, the retailers assigned to a given DC tend to select the same safety stock placement strategy, except for those retailers with higher demands. For these retailers, strategy one tends to be selected as a large retailer tends to take proportionally less benefit from demand variability pooling at a DC (it represents a large share of the pool itself). Furthermore, strategy two tends to be selected when DCs have a large number of retailers assigned, to benefit from demand variability pooling. In Figure 1,a, we observe that relatively long order lead times $\left(L T_{d}\right)$ 

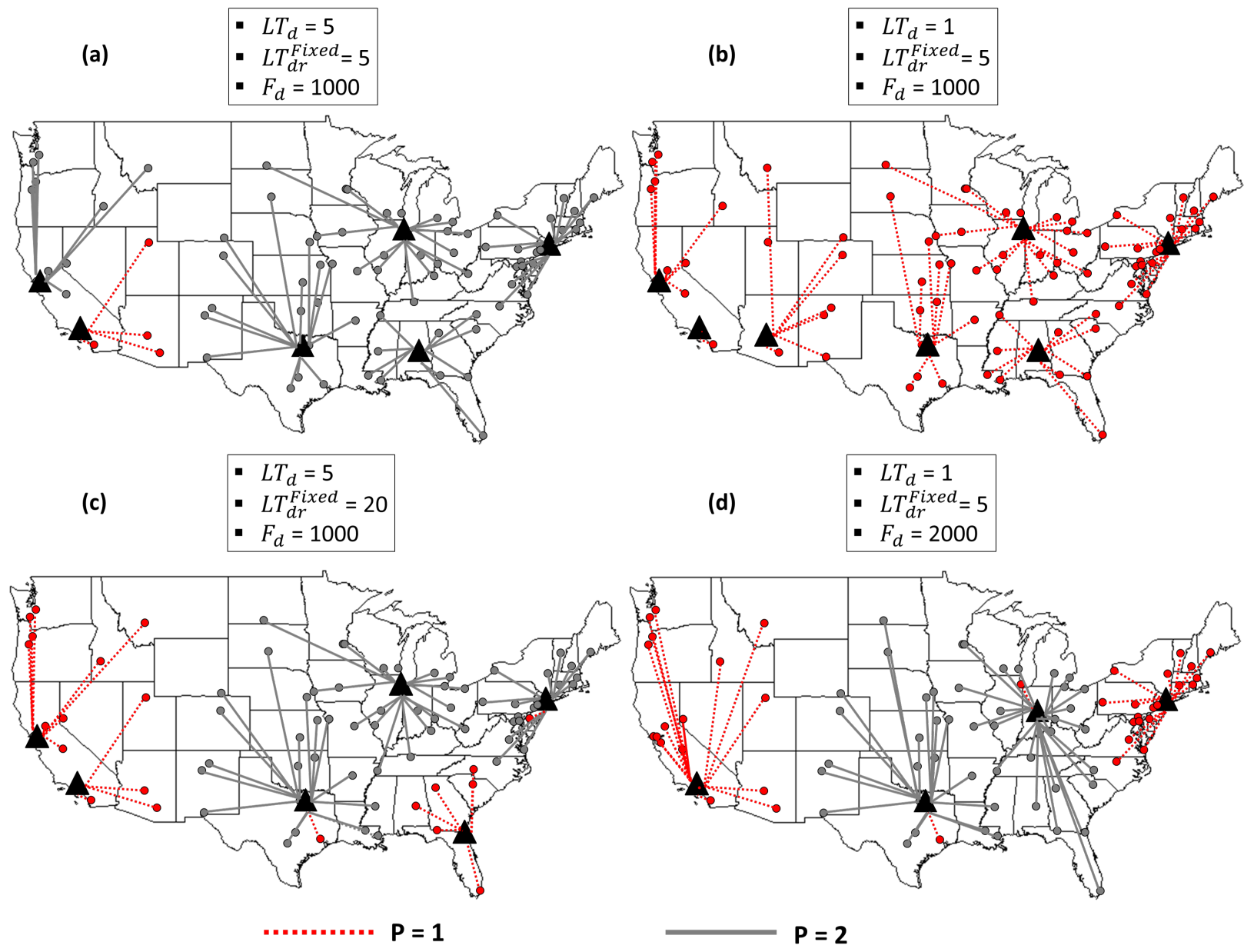

Figure 1: Safety stock placement strategy selected for different values of order lead time $\left(L T_{d}\right)$, lead time between DCs and retailers $\left(L T_{d r}^{\text {Fixed }}\right)$ and facility opening cost $\left(F_{d}\right)$, with $C V_{r}^{E D}=C V_{r}^{R D}=0.8, S_{r}^{\%}=60 \%$, $P_{r}^{E D}=70 \%$ and $T=0.5$.

result in DCs holding safety stocks and a limited number of DCs opened to benefit from demand variability pooling. When the order lead time is reduced in Figure 1,b, the DCs do not hold safety stocks and a larger number of DCs is opened (as DCs are not pooling safety stocks). When comparing Figures 1.a and 1.c, we see that an increase of the lead times between DCs and retailers $\left(L T_{d r}^{\text {Fixed }}\right)$ motivates a broader use of strategy one, and moving the south east DC further east. In Figure 1.d, we show how DCs are centralized when the facility opening costs are increased, leading to four DCs opened. For this case, safety stocks are held at those DCs that serve a larger number of retailers and benefit largely 


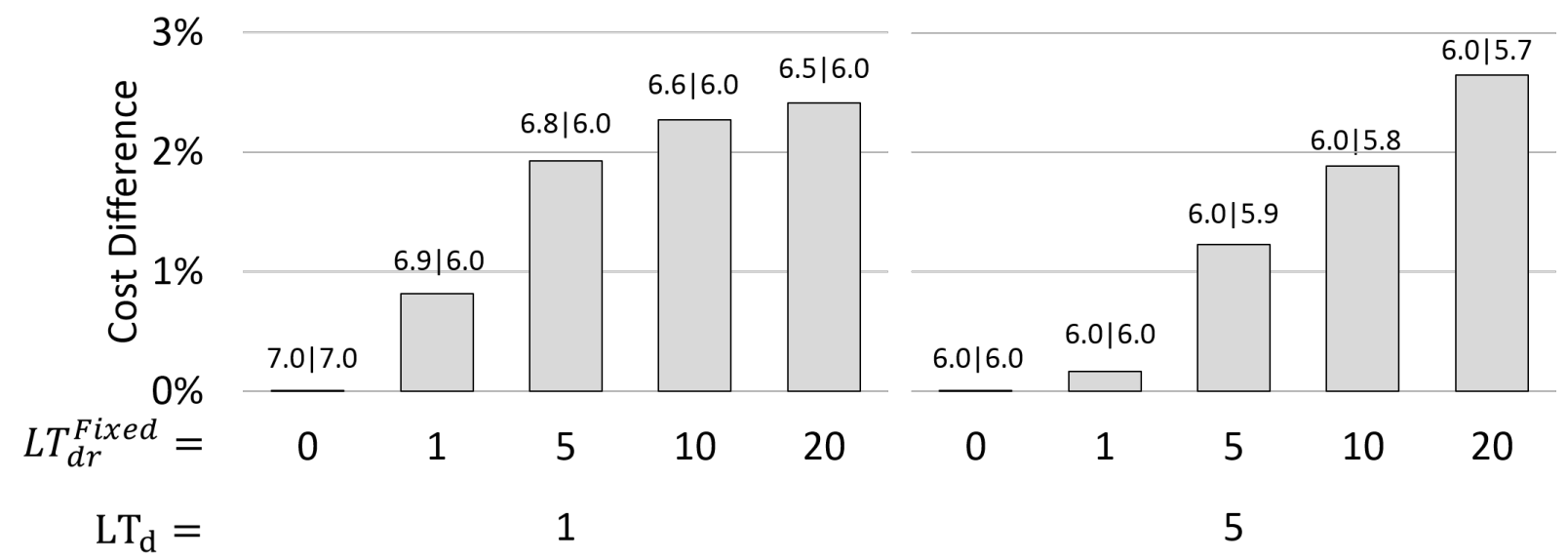

Figure 2: Total cost difference (in percentage) between our model and the model forcing safety stocks to be held at retailers and at DCs, for different lead times between DCs and retailers $\left(L T_{d r}^{\text {Fixed }}\right.$ ) and different order lead times $\left(L T_{d}\right)$. Above each bar, we give the number of DCs opened with our model (left) and with the model forcing safety stocks to be held at DCs (right). The values in the figure are averages over 90 instances (for each value of $C V_{r}^{E D}=C V_{r}^{R D}, S_{r}^{\%}, P_{r}^{E D}$ and $T_{r}$ )

from demand variability pooling.

The previous results show the interdependency between location decisions, lead times, safety stock placement decisions, demand variability pooling and lead time pooling. In this context, Figure 2 helps in quantifying the importance of integrating safety stock placement decisions when designing the supply chain. In Figure 2, we compare the total costs found by our model, where we decide whether to hold safety stocks at DCs or not, with those found by the model that does not integrate the decision and forces DCs to hold safety stocks. For supply chains in which the lead times between DCs and retailers are proportionally long, giving the option to have safety stocks only at retailers can reduce the overall cost by more than $2 \%$. We also see in Figure 2 that the safety stock placement strategy has an impact on the optimal number of facilities. For the model where we force safety stocks to be held at DCs, the number of DCs opened is reduced to benefit from demand variability pooling. Overall, Figure 2 shows that the integration of safety stock placement decisions can lead to significant cost savings and different optimal location decisions.

Another interesting insight arises from the selection of the delivery strategies at retailers. In all the computational experiments, we found that, for each specific instance, all the re- 


\begin{tabular}{|c|c|c|c|c|c|c|c|}
\hline \multirow[b]{2}{*}{$T_{r}$} & \multirow[b]{2}{*}{$L T_{d r}^{\text {Fixed }}$} & \multicolumn{3}{|c|}{$C V_{r}^{E D}=0.4$} & \multicolumn{3}{|c|}{$C V_{r}^{E D}=0.8$} \\
\hline & & $S_{r}^{\%}=20$ & $S_{r}^{\%}=60$ & $S_{r}^{\%}=100$ & $S_{r}^{\%}=20$ & $S_{r}^{\%}=60$ & $S_{r}^{\%}=100$ \\
\hline \multirow{4}{*}{0.05} & 1 & ED & ED & ED & ED & $\mathrm{RD}$ & $\mathrm{RD}$ \\
\hline & 5 & ED & $\mathrm{RD}$ & $\mathrm{RD}$ & $\mathrm{RD}$ & $\mathrm{RD}$ & $\mathrm{RD}$ \\
\hline & 10 & $\mathrm{RD}$ & $\mathrm{RD}$ & $\mathrm{RD}$ & $\mathrm{RD}$ & $\mathrm{RD}$ & $\mathrm{RD}$ \\
\hline & 20 & $\mathrm{RD}$ & $\mathrm{RD}$ & $\mathrm{RD}$ & $\mathrm{RD}$ & $\mathrm{RD}$ & $\mathrm{RD}$ \\
\hline \multirow{4}{*}{0.25} & 1 & ED & $\mathrm{ED}$ & ED & ED & ED & $\mathrm{ED}$ \\
\hline & 5 & $\mathrm{ED}$ & $\mathrm{ED}$ & ED & ED & ED & ED \\
\hline & 10 & ED & ED & ED & ED & ED & $\mathrm{RD}$ \\
\hline & 20 & ED & ED & ED & ED & $\mathrm{RD}$ & $\mathrm{RD}$ \\
\hline
\end{tabular}

Table 5: Express deliveries (ED) and regular deliveries (RD) applied for different coefficients of variation $\left(C V_{r}^{E D}=C V_{r}^{R D}\right)$, fee $\left(T_{r}\right)$, service times $\left(S_{r}^{\%}\right)$ and lead times between DCs and retailers $\left(L T_{d r}^{\text {Fixed }}\right)$. The values in the table are found over 10 instances (we get the same delivery strategy for each value of $L T_{d}$ and $\left.P_{r}^{E D}\right)$.

tailers apply the same delivery strategy. Table 5 shows in which situations express deliveries (ED) or only regular deliveries (RD) are offered. Firstly, with longer service times $\left(S_{r}^{\%}\right)$, safety stocks are reduced for regular deliveries, and retailers thus tend to select this type of delivery. Secondly, we observe in Table 5 that increasing the demand uncertainty $\left(C V_{r}^{E D}\right)$ and the lead time between DCs and the retailers $\left(L T_{d r}^{\text {Fixed }}\right)$ leads to the selection of only regular deliveries in order to mitigate the safety stock cost increase. Finally, by increasing the fee $\left(T_{r}\right)$, the retailers have incentives to improve the customer's service, and tend to offer express deliveries.

A classic trade-off in the supply chain design problem is whether to centralize facilities in order to gain economies of scale, or to decentralize them to become more responsive by being closer to retailers. One interesting result from our model is that the service times of regular deliveries may impact this trade-off, and the location and the number of DCs opened. This is illustrated in Figure 3 , for two instances. We see that safety stock placement strategy one is selected in both cases, so that DCs do not hold safety stocks. Regarding the delivery strategies, the increase in service time leads to drop express deliveries as they become comparatively more expensive. Finally, and most interestingly, we observe in Figure 3 that, when DCs do not hold safety stocks, longer service times for regular deliveries may 

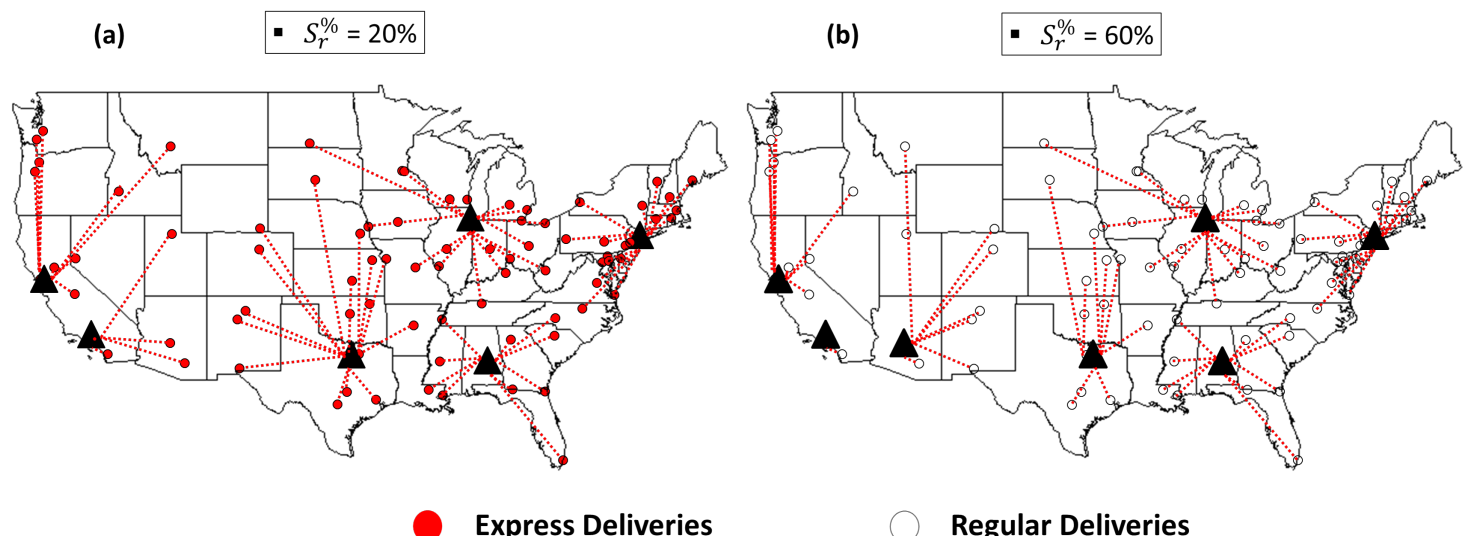

Regular Deliveries

Figure 3: Safety stock placement $(p=1)$ and delivery strategy selected for different service times $\left(S_{r}^{\%}\right)$, with $L T_{d}=1, L T_{d r}^{\text {Fixed }}=5, C V_{r}^{E D}=C V_{r}^{R D}=0.4, P_{r}^{E D}=30 \%$ and $T_{r}=0.05$.

lead to an increase on the number of DCs opened, which reduces the lead times between DCs and retailers. Due to the concavity of the square root function (see the first term of Equation 6), a decrease of these lead times $\left(L T_{d r}\right)$ has a larger impact when the net lead times are short. Longer service times may thus encourage to reduce lead times and increase the number of DCs. We can conclude from Figure 3 that the integration of delivery strategies in the supply chain design problem is justified as it may impact the number and location of DCs.

\section{Conclusions}

This paper presents an approach for integrating safety stock placement and delivery strategy decisions into the strategic supply chain design. We propose a mathematical model that considers differentiated service times and two different classes of customers regarding their delivery time preferences. Our model minimizes the costs of transportation, facility opening, cycle inventory, ordering and safety stocks. The resulting non-linear model is formulated as a conic quadratic mixed-integer program that can be solved to optimality in reasonable computational time using standard optimization software packages.

Managerial insights are then inferred from the computational experiments. We discuss the trade-off between demand variability pooling and lead time pooling. In particular, 
proportionally short lead times between DCs and retailers result in a supply chain where DCs and retailers tend to hold safety stocks in order to benefit from pooling demand variability at DCs. In such a supply chain, the retailers tend to offer express deliveries to their customers, except if the service times are long or if the fees are very small. When proportionally long lead times between DCs and retailers are considered, the supply chain is configured to benefit from lead time pooling so that DCs do not hold safety stocks. In this case, the retailers only propose regular deliveries, except if the service times are short or if the fees are large. We also show the impact of demand uncertainty on safety stock placement decisions. The retailers with higher demands tend to hold safety stocks and to not pool demand variability at DCs. Moreover, the DCs serving a large number of retailers tend to hold safety stocks to benefit from pooling demand variability at DCs. Finally, we discuss the impact of service times on the number of DCs opened. When lead time pooling strategies are followed, the number of DCs opened increases. The integration of safety stock placement and delivery decisions into the strategic design of supply chains is justified as we have shown that these decisions and the location decisions are interrelated and as it allows to balance all the aforementioned trade-offs and reduce the overall supply chain cost.

In the future, this research could be extended by integrating other features such as correlated demands, three stage supply chains, direct shipments from the production sites to the retailers, capacity constraints at retailers and DCs, or the consideration of more than two customer classes. We could also consider a more precise customer behaviour modelling approach with respect to the relation between the fee and the delivery times, including a penalty when express deliveries' demands are not proposed. Features such as multiple modes of transportation, capacity constraints at retailers and at DCs, or multiple products could also be considered.

\section{References}

Atamtürk, A., Berenguer, G., Shen, Z.-J. M., 2012. A conic integer programming approach to stochastic joint location-inventory problems. Operations Research, 60 (2), 366-381. 
Atamtürk, A., Narayanan, V., 2008. Polymatroids and mean-risk minimization in discrete optimization. Operations Research Letters, 36 (5), 618-622.

Beamon, B. M., 1998. Supply chain design and analysis: Models and methods. International Journal of Production Economics, 55 (3), 281-294.

Bravo, J. J., Vidal, C. J., 2013. Freight transportation function in supply chain optimization models: A critical review of recent trends. Expert Systems with Applications, 40 (17), 6742-6757.

Chen, H., Li, P., 2015. Optimization of (R, Q) policies for serial inventory systems using the guaranteed service approach. Computers \& Industrial Engineering, 80, 261-273.

Chopra, S., Meindl, P., 2016. Supply Chain Management: strategy, planning, and operation, 6th Edition.

Daskin, M., Coullard, C., Shen, Z.-J., 2002. An inventory-location model: Formulation, solution algorithm and computational results. Annals of Operations Research, 110 (1-4), 83-106.

Daskin, M. S., 1995. Network and discrete location: Models, algorithms, and applications. Wiley, New York.

Edmonds, J., 1970. Submodular functions, matroids, and certain polyhedra. Combinatorial Structures and Their Applications, 69-87.

Eppen, G. D., 1979. Effects of centralization on expected costs in a multi-location newsboy problem. Management Science, 25 (5), 498-501.

Eruguz, A. S., Sahin, E., Jemai, Z., Dallery, Y., 2016. A comprehensive survey of guaranteed-service models for multi-echelon inventory optimization. International Journal of Production Economics, 172, 110-125.

Evers, P. T., 1995. Expanding the square root law: An analysis of both safety an. Logistics and Transportation Review, 31 (1), 1.

Farahani, R. Z., Rashidi Bajgan, H., Fahimnia, B., Kaviani, M., 2015. Location-inventory problem in supply chains: a modelling review. International Journal of Production Research, 53 (12), 3769-3788.

Funaki, K., 2012. Strategic safety stock placement in supply chain design with due-date based demand. International Journal of Production Economics, 135 (1), 4-13.

Grahl, J., Minner, S., Dittmar, D., 2014. Meta-heuristics for placing strategic safety stock in multi-echelon inventory with differentiated service times. Annals of Operations Research, 1-16.

Graves, S. C., Willems, S. P., 2003. Supply chain design: safety stock placement and supply chain configuration. Vol. 11. Elsevier, pp. 95-132.

Graves, S. C., Willems, S. P., 2005. Optimizing the supply chain configuration for new products. Management Science, 51 (8), 1165-1180.

Gunasekaran, A., Patel, C., McGaughey, R. E., 2004. A framework for supply chain performance measurement. International Journal of Production Economics, 87 (3), 333-347.

Hua, N. G., Willems, S. P., 2015. Analytical insights into two-stage serial line supply chain safety stock. International Journal of Production Economics. 
Humair, S., Ruark, J. D., Tomlin, B., Willems, S. P., 2013. Incorporating stochastic lead times into the guaranteed service model of safety stock optimization. Interfaces, 43 (5), 421-434.

Inderfurth, K., Minner, S., 1998. Safety stocks in multi-stage inventory systems under different service measures. European Journal of Operational Research, 106 (1), 57-73.

Klose, A., Drexl, A., 2005. Facility location models for distribution system design. European Journal of Operational Research, 162 (1), 4-29.

Kumar, S., Tiwari, M., 2013. Supply chain system design integrated with risk pooling. Computers \& Industrial Engineering, 64 (2), 580-588.

Li, H., Jiang, D., 2012. New model and heuristics for safety stock placement in general acyclic supply chain networks. Computers \& Operations Research, 39 (7), 1333-1344.

Magnanti, T. L., Shen, Z.-J. M., Shu, J., Simchi-Levi, D., Teo, C.-P., 2006. Inventory placement in acyclic supply chain networks. Operations Research Letters, 34 (2), 228-238.

Melo, M. T., Nickel, S., Saldanha-Da-Gama, F., 2009. Facility location and supply chain management-a review. European Journal of Operational Research, 196 (2), 401-412.

Minner, S., 2000. Strategic safety stocks in supply chains. Springer.

Miranda, P. A., Garrido, R. A., 2004. Incorporating inventory control decisions into a strategic distribution network design model with stochastic demand. Transportation Research Part E: Logistics and Transportation Review, 40 (3), 183-207.

Moncayo-Martínez, L. A., Zhang, D. Z., 2013. Optimising safety stock placement and lead time in an assembly supply chain using bi-objective max-min ant system. International Journal of Production Economics, $145(1), 18-28$.

Mula, J., Peidro, D., Díaz-Madroñero, M., Vicens, E., 2010. Mathematical programming models for supply chain production and transport planning. European Journal of Operational Research, 204 (3), 377-390.

Novich, N., 1990. Distribution strategy: Are you thinking small enough?. Sloan Management Review, 32 (1), 71-77.

Osman, H., Demirli, K., 2012. Integrated safety stock optimization for multiple sourced stockpoints facing variable demand and lead time. International Journal of Production Economics, 135 (1), 299-307.

Owen, S. H., Daskin, M. S., 1998. Strategic facility location: A review. European Journal of Operational Research, 111 (3), 423-447.

Park, S., Lee, T.-E., Sung, C., 2010. A three-level supply chain network design model with risk-pooling and lead times. Transportation Research Part E: Logistics and Transportation Review 46 (5), 563-581.

Ray, S., Jewkes, E. M., 2004. Customer lead time management when both demand and price are lead time sensitive. European Journal of operational research 153 (3), 769-781.

ReVelle, C. S., Eiselt, H. A., 2005. Location analysis: A synthesis and survey. European Journal of Opera- 
tional Research, 165 (1), 1-19.

Rodriguez, M. A., Vecchietti, A. R., Harjunkoski, I., Grossmann, I. E., 2014. Optimal supply chain design and management over a multi-period horizon under demand uncertainty. Part I: MINLP and MILP models. Computers \& Chemical Engineering, 62, 194-210.

Schuster, M., Tancrez, J.-S., 2016. A heuristic algorithm for solving large location-inventory problems with demand uncertainty. Working Paper, Université catholique de Louvain.

Shahabi, M., Akbarinasaji, S., Unnikrishnan, A., James, R., 2013. Integrated inventory control and facility location decisions in a multi-echelon supply chain network with hubs. Networks and Spatial Economics, $13(4), 497-514$.

Shen, Z.-J. M., 2007. Integrated supply chain design models: a survey and future research directions. Journal of Industrial and Management Optimization, 3 (1), 1-27.

Shen, Z.-J. M., Coullard, C. R., Daskin, M. S., 2003. A joint location-inventory model. Transportation Science, 37 (1), 40-55.

Simpson, K. F., 1958. In-process inventories. Operations Research, 6 (6), 863-873.

Smykay, E. W., 1973. Physical distribution management.

Sourirajan, K., Ozsen, L., Uzsoy, R., 2009. A genetic algorithm for a single product network design model with lead time and safety stock considerations. European Journal of Operational Research, 197 (2), 599 608.

Teimoury, E., Modarres, M., Monfared, A. K., Fathi, M., 2011. Price, delivery time, and capacity decisions in an $\mathrm{m} / \mathrm{m} / 1$ make-to-order/service system with segmented market. The International Journal of Advanced Manufacturing Technology 57 (1-4), 235-244.

You, F., Grossmann, I. E., 2010. Integrated multi-echelon supply chain design with inventories under uncertainty: MINLP models, computational strategies. AIChE Journal, 56 (2), 419-440. 